\title{
Young's Modulus and Poisson's Ratio of Polyurethane Adhesive in Lightweight Floor System
}

\author{
Jacek Karpiesiuk* \\ Member of the Polish Chamber of Civil Engineers, Bialystok University of Technology, Poland
}

*Corresponding author: Jacek Karpiesiuk, Bialystok University of Technology, Government Building Expert in construction and building specialties, Member of the Polish Chamber of Civil Engineers, Wiejska str. 45A, 15-351 Bialystok, Poland

\begin{abstract}
The Lightweight Floor System with aluminum foil (LFS-L) has high thermal efficiency and meets the conditions of comfortable use. With this in mind, research work in the field of civil engineering began, which resulted in the development of an original lightweight technical solution for the heating floor. The layer connecting floor tiles with aluminum foil, which dissipates heat in LFS-L is polyurethane adhesive. To develop a computational model for a lightweight floor without screeds was appointed the strength indicators of this adhesive, such as Young's modulus and Poisson's ratio, which are not available in the literature. They were determined using tensile strength tests of polyurethane adhesive.
\end{abstract}

Keywords: Polyurethane adhesive; Lightweight floor system; Young's modulus; Poisson's ratio

\section{Introduction}

Monography [1] confirms that Lightweight Floor System with aluminum foil, abbreviated as LFS-L meets at least three assumed hypotheses:

a. Has high thermal efficiency and meets the conditions of comfortable use with a heating coil spacing up to $20 \mathrm{~cm}$, with the so-called „metal lamellas”.

b. Low thermal inertia LFS-L makes it the most efficient of the existing solutions on the market, which confirms its high usability in devices using renewable energy sources (RES).

c. A light underfloor heating system with metal lamellas is the most advantageous solution when we subject it to MultiCriteria Decision Analysis Method (MCDA).

The construction of the Lightweight Floor System (LFS) without screeds, with a heating coil, consists of several layers. When mounting this system on the floor, the bottom layer is usually made of extruded XPS polystyrene or expanded EPS boards. They are available in raw or covered with the so-called "metal lamellas" or aluminum foil (LFS-L). Insulation boards are grooved for the placement of heating pipes, most often supplied with water. Concrete surfaces are not laid, and the insulation boards are covered with an adhesive. Standard [2] is dedicated to this system with a heating coil mounted on metal heat dissipators. An easier technical solution that does not take time to prime and strengthen of the metal lamellas when gluing tiles over, is the use of polyurethane adhesives. This adhesive is most important in the whole crosssection of a light floor without screeds, because it must transfer various types of internal stresses arising from external loads, including thermal interactions between layers with significantly different strength indicators. Most of them are available in the literature. There is only a lack of data on polyurethane adhesives connecting ceramic tiles with lamellas, such as Young's modulus E, Poisson's ratio $v$. To determine them, tensile tests were carried out in the laboratory of the Bialystok University of Technology.

\section{Research Methodology}

In the case of the tensile strength, a research was performed using samples of polyurethane adhesive. After analyzing the literature, including the monography [3], it was decided to make paddle samples of polyurethane adhesive as those which guarantee the final determination of the necessary data, i.e. Young's modulus and Poisson's ratio in axial tensile test. For this purpose, four samples of Sika BondT8 adhesives were prepared, as shown in (Figure 1). The test used a dynamic two-axle testing machine MTS 858 Mini Bionix with a hydraulic drive, mounted on a 359-load frame with a range of axial loads $\pm 25 \mathrm{kN}$. This machine has cooperated with the ARAMIS 3D 4M vision system, using the method of Digital Image Correlation (DIC), as showed in (Figure 2). Dependence of longitudinal deformation $\varepsilon_{m}$ on tension stress was tested on four 
samples. A set of DIC system devices is used for non-contact, threedimensional measurements of deformations caused by static and dynamic loads. The task of this method is to analyze, calculate displacements and document material deformations. Graphic representation of the obtained test results gives the opportunity to fully understand the behavior of the tested object.

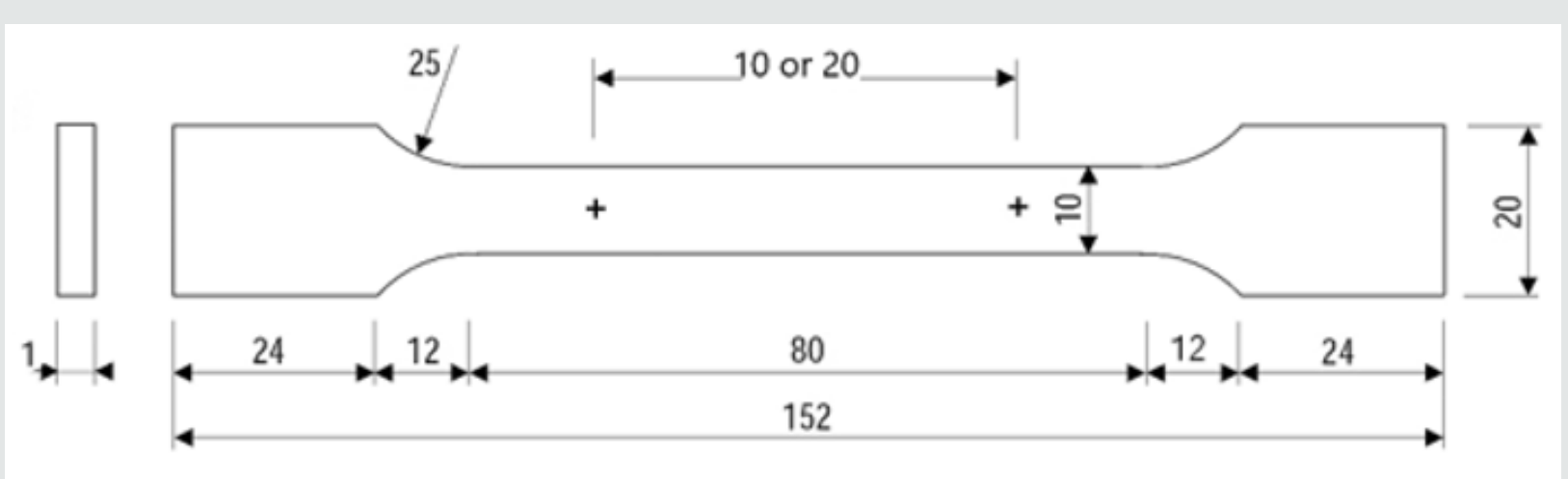

Figure 1: The paddle samples with dimensions according to EN ISO 527-2 standard, with 10- or 20mm measuring base.

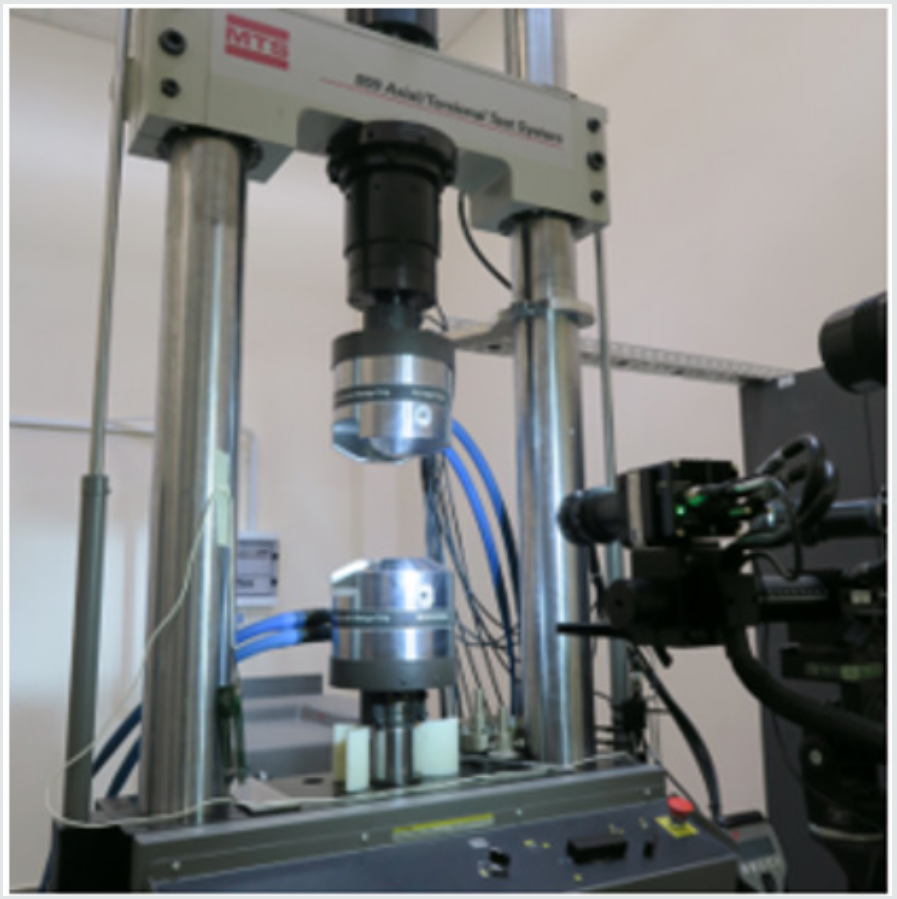

Figure 2: Measurement of $\varepsilon_{\mathrm{m}}$ deformation using the ARAMIS 3D 4M vision system.

\section{The Research Results}

The main purpose of the research was to determine Young's modulus E and Poisson's ratio $v$ of the polyurethane adhesive tested on previously prepared paddle samples. To determine the material stress, the calculation formula (1) was used:

$$
\sigma=\frac{F}{A}
$$

$\sigma$ - polyurethane stress [MPa], F-tension force[N], A- the crosssectional area of the sample $\left[\mathrm{mm}^{2}\right]$

Based on formula (1), the quadratic functions characterizing the relationship of stress $\sigma$ to longitudinal deformation $\varepsilon_{m}$ at the tension strength of polyurethane adhesive were determined. The dependence of deformation $\varepsilon_{m}$ on tensile stress in MPa is presented in (Figure 3-6). Summary drawing of four designated functions showing the dependence of the longitudinal strain $\varepsilon_{m}$ on the tensile stress $\sigma$ in MPa of each sample is in (Figure 7). The elasticity of different materials depends on two factors - the density and stiffness of interatomic bonds. The smaller and stronger bonded the structural atoms of the material are, the greater its rigidity. Steel and polymers can serve as a comparative example. When the density of atoms and their bonds differ, they affect the stiffness of the material.

Young's modulus of longitudinal elasticity was determined on the basis of formula (2):

$$
E=\frac{\Delta \sigma}{\Delta E_{s p}}
$$




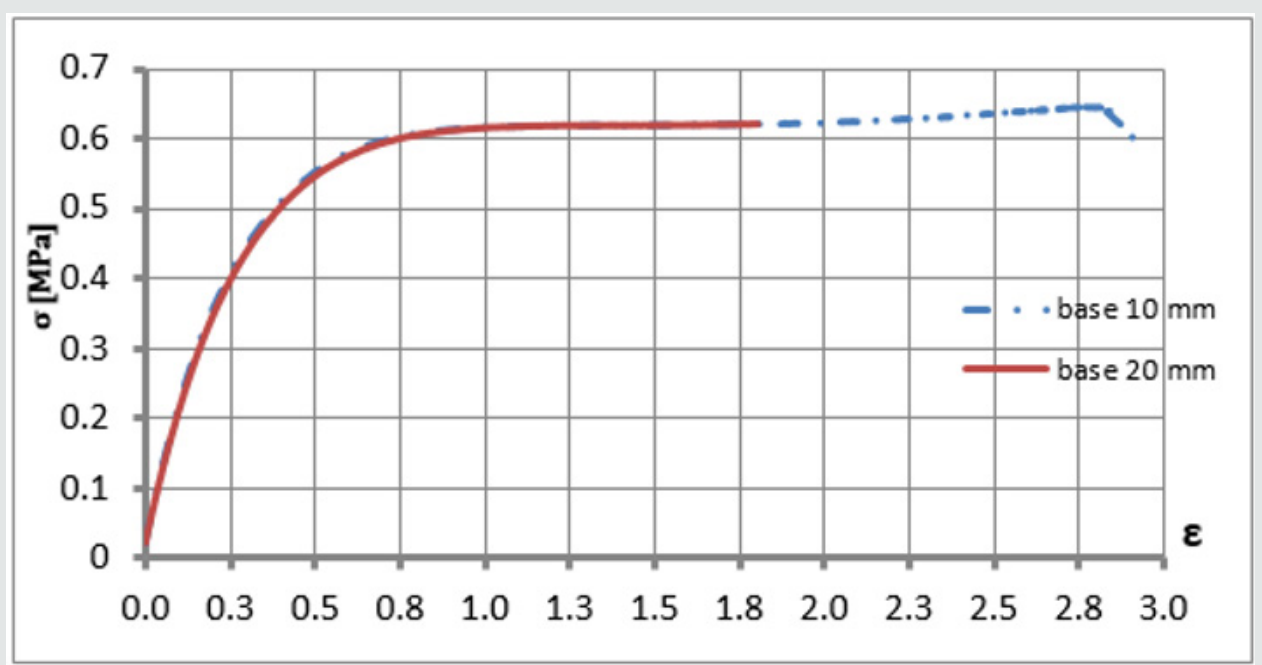

Figure 3: Dependence $\sigma$ on $\varepsilon$ of polyurethane adhesive, sample PUR_1 under tension force, for 10- and 20-mm measuring base.

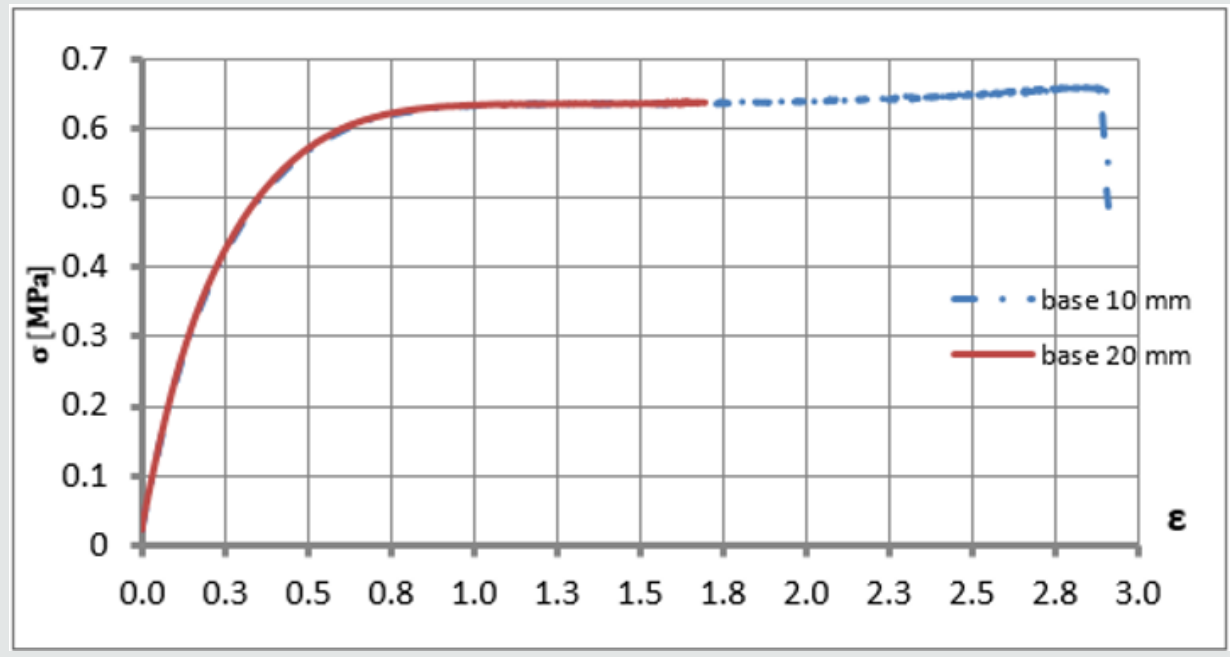

Figure 4: Dependence $\sigma$ on $\varepsilon$ of polyurethane adhesive, sample PUR_2 under tension force, for 10- and 20mm measuring base.

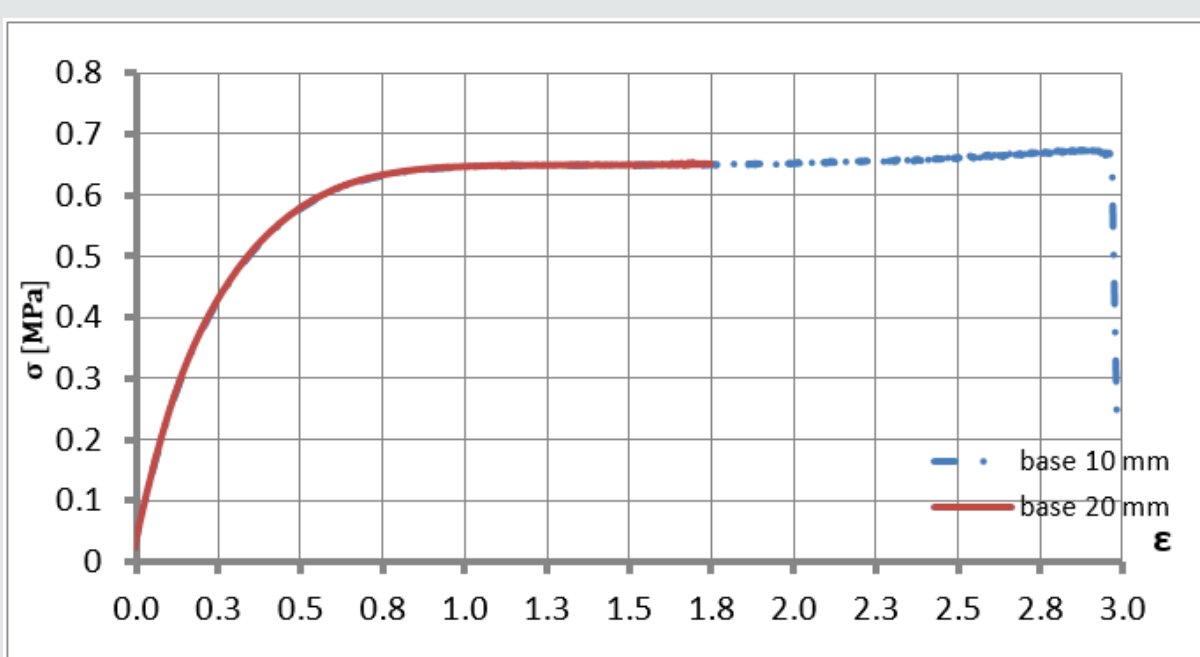

Figure 5: Dependence $\sigma$ on $\mathcal{E}$ of polyurethane adhesive, sample PUR_3 under tension force, for 10 and 20 mm, measuring base. 


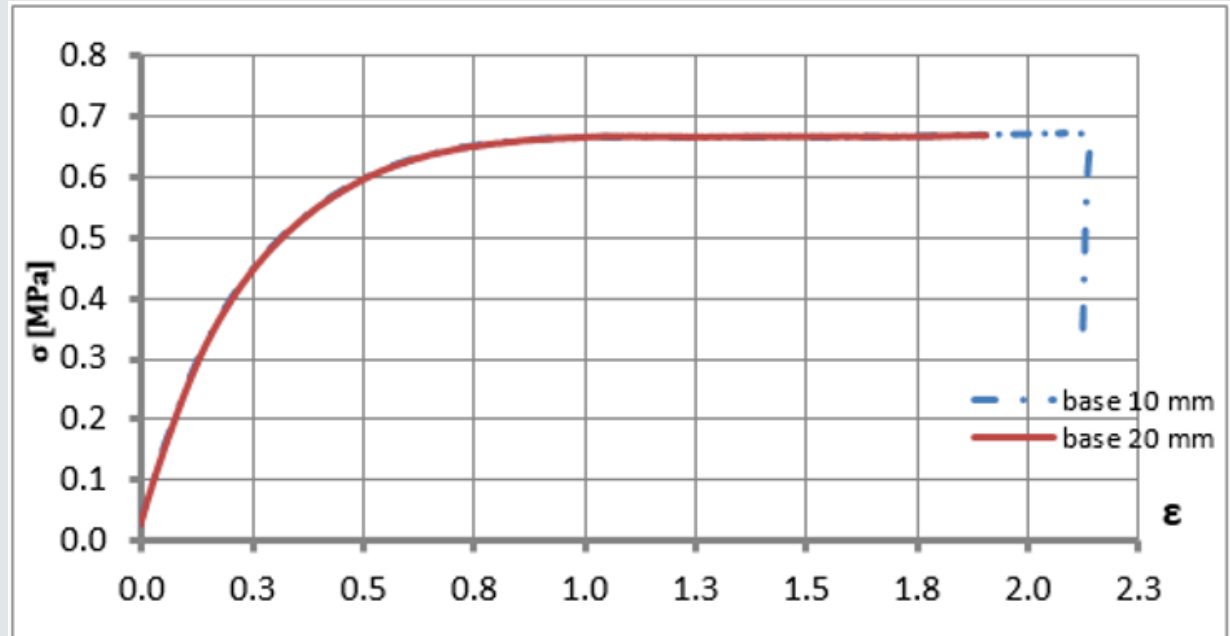

Figure 6: Dependence $\sigma$ on $\mathcal{E}$ of polyurethane adhesive, sample PUR_4 under tension force, for 10- and 20-mm measuring base.

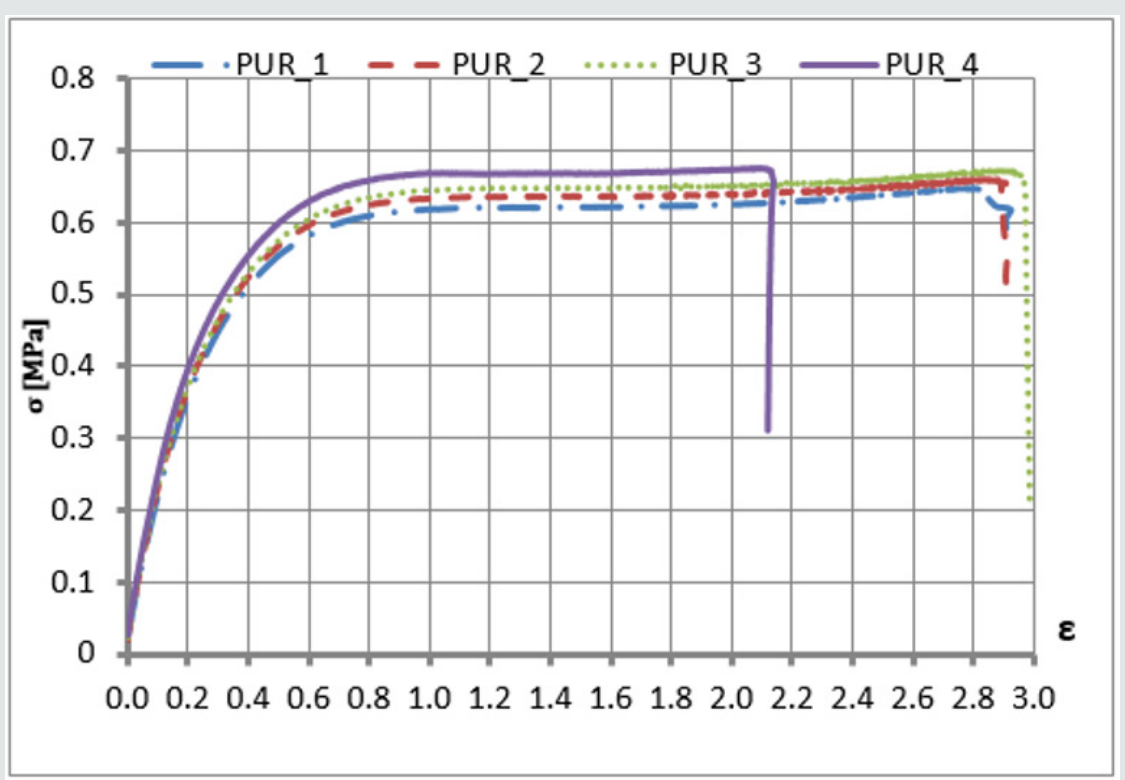

Figure 7: Summary dependence $\sigma$ on $\varepsilon$ of polyurethane adhesive, all PUR samples under tension force, for $10 \mathrm{~mm}$ measuring base.

\section{E- Young's modulus}

$\Delta \sigma$-a difference of material stress in the elastic phase

$\Delta \varepsilon_{\mathrm{sp}}$-a difference of material deformations in the elastic phase

For calculations, $\Delta \sigma=0.2-0,1=0.1 \mathrm{MPa}$ was used in all samples, and in this range the corresponding deformations, $\Delta \varepsilon_{\mathrm{sp}}$-a difference of material deformations in the elastic phase. The formula (3) was used to determine the Poisson's ratio. At the same time, the same stress range was taken as when calculating Young's modulus.

$v=\frac{\Delta \varepsilon_{n}}{\Delta \sigma} \cdot \frac{\Delta \sigma}{\Delta \varepsilon_{m}}$

$v$ - Poisson's ratio,

\section{$\Delta \varepsilon_{n}$ - transverse deformation \\ $\Delta \varepsilon_{m}$ - longitudinal deformation \\ $\Delta \sigma=0.2-0.1=0.1[\mathrm{MPa}]$}

The strength average and individual samples indicators calculated from the formulas (2) and (3) are given in Table 1. In addition, the table provides information on the maximum tensile stress $\sigma_{t \text { max }}$ (for $10 \mathrm{~mm}$ measuring base) with the related longitudinal deformations $\varepsilon_{m}$ and transverse deformations $\varepsilon_{n}$ from the experiment: $\sigma_{t \max }-$ maximum tensile stress; $\varepsilon_{m}$-longitudinal tensie deformation; $\varepsilon_{n}$ - transverse tensile deformation. $E$ - Young's modulus of polyurethane adhesive; $E_{\text {avg }}$-average Young's modulus of polyurethane adhesive, $v$ - Poisson's ratio of polyurethane adhesive; $v_{\text {avg }}$ - average Poisson's ratio of polyurethane adhesive. 
Table 1: Maximum tensile stresses, deformations and strength indexes $E$ and $v$ in the tested polyurethane adhesive Sika Bond T8.

\begin{tabular}{|c|c|c|c|c|c|c|c|}
\hline No of Sample & $\boldsymbol{\sigma}_{\text {tmax }}[\mathrm{MPa}]$ & $\boldsymbol{\varepsilon}_{\mathrm{m}}[-]$ & $\boldsymbol{\varepsilon}_{\mathrm{n}}[-]$ & $\mathbf{E}[\mathbf{M P a}]$ & $\mathbf{E}_{\text {avg }}$ & $\mathbf{v}[-]$ & $\boldsymbol{v}_{\text {avg }}[-]$ \\
\hline PUR_1 & 0.62 & 1.63 & 0.35 & 1.9 & 2.1 & 0.42 & 0.41 \\
\hline PUR_2 & 0.63 & 1.28 & 0.3 & 2.08 & & 0.41 \\
\hline PUR_3 & 0.65 & 1.74 & 0.34 & 2.08 & & 0.4 & \\
\hline PUR_4 & 0.67 & 1 & 0.27 & 2.13 & & 0,42 & \\
\hline
\end{tabular}

Due to the extremely high elasticity of the polyurethane adhesive at low stress of the cylindrical samples, used in the compressive strength test [4], it was recommended to perform a tensile strength test of the polyurethane adhesive, at the actual thickness of its use, 1-2 mm. The results of determining Young's modulus and Poisson's ratio of the Sika BondT8 polyurethane adhesive in [4] were as follows $-E_{p}=2.5 \mathrm{MPa}, v_{\text {avg }}=0.32$. This confirms the correctness of Young's modulus determination, equal $E_{\text {avg }}=2.1 \mathrm{MPa}$ (the results are not greater than $20 \%$, which is within the coefficient of variation of polyurethane adhesives [3]), and the Poisson's ratio should be taken as correct in accordance with the tensile strength test, i.e. $\mathbf{v}_{\text {avg }}=0.41$. The reason for this is that the thickness of the polyurethane adhesive adopted in this test reflecting the actual working conditions at the construction site (in the LFS-L).

\section{Conclusion}

Tensile strength tests of the paddle samples allowed the determination of Sika BondT8 polyurethane adhesive strength indexes, specifically Young's modulus, and Poisson's ratio. It was possible using Digital Image Correlation in the Aramis system. This type of research confirmed the correctness previous results of Young's modulus, obtained thanks to the compressive strength test of cylindrical samples, using the extensometers and digital measurement methods and have given corrections for Poisson's ratio. In this way, the recommendations performing a tensile strength test of the polyurethane adhesive, at the actual thickness of its use 1-2 mm have fulfilled. The experiments additionally determined the maximum tensile stresses and longitudinal, and transverse deformations of polyurethane glue Sika BondT8. With this data, they can be used in numerical calculations of a Lightweight Floor System with lamellas (LFS-L) with a heating coil in the Finite Element Method, as well as for all other calculations needed.

\section{Acknowledgment}

The author of the article would like to thank Mr. Wojciech Grodzki from the Bialystok University of Technology for technical cooperation. The research was carried out as part of my own work from Bialystok University of Technology No. W/WBIIŚ/15/2019, financed from the subsidy provided by the Ministry of Science and Higher Education.

\section{References}

1. Karpiesiuk J (2019) Thermal Efficiency of Lightweight Radiant Heating System. Monography (in Polish). GlobeEdit pp. S-140.

2. (2001) Floor heating systems. Design and type testing of waterborne heat systems for lightweight structures. NORDTEST.

3. Zajac B (2018) Shear, rigid and flexible glued joints operating at elevated temperature. Monography (in Polish), Cracow University of Technology. Civil Engineering Series pp. S-170.

4. Karpiesiuk J (2020) Mechanical Strength Indicators of Polyurethane Adhesive in Lightweight Floor System. (in revision).

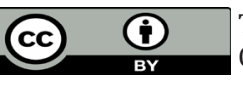

This work is licensed under Creative Commons Attribution 4.0 License

To Submit Your Article Click Here: Submit Article
DOI: 10.32474 /MAMS.2020.02.000139

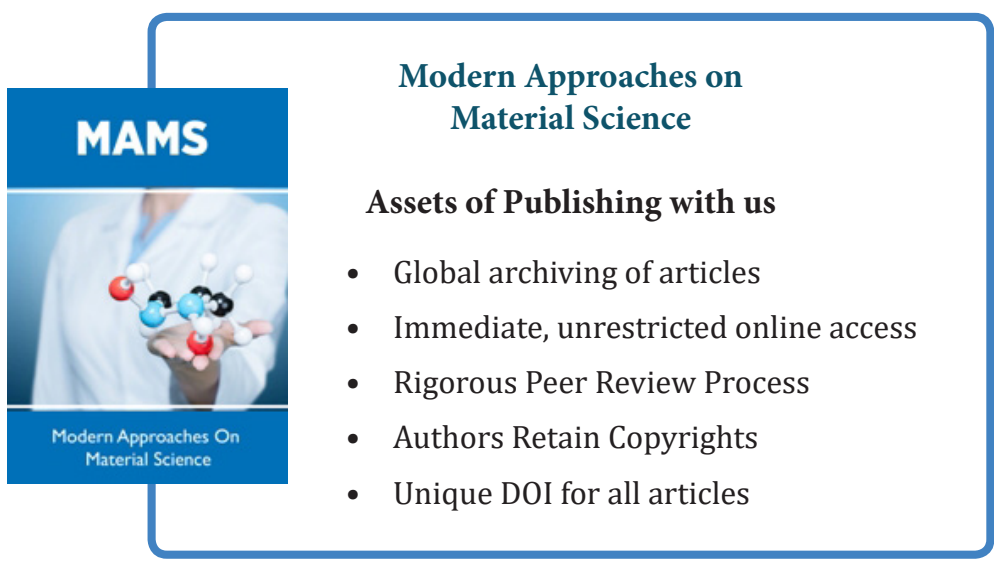

